\begin{tabular}{c} 
International Journal of Engineering \& Technology, $7(3.31)(2018) 25-29$ \\
International Journal of Engineering \& Technology \\
SPC \\
Website: $w$ ww.sciencepubco.com/index.php/IJET \\
Research paper \\
\hline
\end{tabular}

\title{
A New Watermarking Scheme for Medical Images with Patient's Details
}

\author{
G. Nagaraju ${ }^{1 *}$, P. Pardhasaradhi ${ }^{2}$, V. S. Ghali ${ }^{3}$ \\ ${ }^{I}$ Department of Electronics and Communications Engineering, SRKR Engineering College, Bhimavaram, \\ Andhra Pradesh, India -534204 \\ ${ }^{2,3} A L R C-R \& D$, Department of Electronics and Communications Engineering, KoneruLakshmaiah Education Foundation, \\ Vaddeswaram, Andhra Pradesh, India -522502 \\ *Corresponding author E-mail:bhanu.raj.nikhil@gmail.com; Tel:+91-9010133466
}

\begin{abstract}
A brain tumor is a mass of cells in your brain that are not normal.Some brain tumors contain cancer and others don't: Brain tumor include both, benign and malignant forms. Benign brain tumors don't have cancer cells. Malignant brain tumors have cancer cells. Differentiating malignant and benign cases is a hard task even for experienced specialists. This work presents how to extract the characteristics and features of tumor image by general segmentation methods for malignant risk computation and presents the use of digital watermarking for applications of automated tumor image analysis. Here personal information such as name, age, gender, location, ADHAAR number, contact number etc., and tumor information such as tumor types, area of the tumor, severity, and any other useful information are embedded to the tumor image. Encrypting that image with well-known encryption algorithms is also possible to avoid unnecessary nuisance from information hackers.
\end{abstract}

Keywords:brain tumor; segmentation; k-means clustering; digital watermarking.

\section{Introduction}

In brain when abnormal cells are increased then tumor occurs. Malignant and benign tumors are mainly two types of brain tumors. Malignant tumors are divided into primary tumors and secondary tumors. Primary tumors are the tumors begin within the brain, and secondary tumors that are spread somewhere else in the body. Generally it is not possible to know the exact reason for most of the brain tumors. The most common types of primary tumors in adults are usually benign. In children, the most common type is a malignant. These tumors are usually diagnosed by medical examination like computed tomography or magnetic resonance imaging. So it is important to extract different features of tumor image. Similarly, storing of this information with patient's details for years is also important for future diagnosis.

Medical imaging plays a important role in the diagnosis of different brain tumors. Nowadays the imaging methods are non-noisy, better resolution techniques, especially computed tomography (CT) and magnetic resonance imaging (MRI) scans. By extracting the features of segmented tumor image, diagnosis of brain tumor can be confirmed. Tumor tissue samples are obtained by histological examination of segmented image.

Image segmentation is the process of partitioning a digital image into multiple segments known as super-pixels. The goal of segmentation is to simplify and/or change the representation of an image into something that is more meaningful and easier to analyze. Image segmentation is mainly based on objects and boundaries. Edge detection is a major part of segmentation process. Practical applications of image segmentation in medical imaging are usually including volume rendered images from computed tomog- raphy and magnetic resonance imaging which are used to locate tumors and volume of measure tissues, study of objective structure, for different surgery planning, simulation of virtual surgery. Different general-purpose segmentation algorithms and techniques have been developed for image segmentation.

A digital watermark is used to mark the different signals such as an audio, video, or image data. Hiding digital data in a digital image is a simple technique using pixel value replacement method. The data which is to be hidden is converted into bits, and these bits are embedded into the image pixels. Both steganography and digital watermarking employ steganographic techniques, while steganography aims for hiding something in signal which is undetectable to human senses, digital watermarking tries to mark something in signal to control the robustness as top priority. Copyright protection, broadcast monitoring, source tracking, and video authentication are different applications of digital watermarking. For removing the digital watermark and replacing the original image, a reversible data hiding technique is used which enables images to be authenticated and restores their original form.

Xinpeng Zhang, presented paper on data hiding technique in encrypted images, this work proposes a new scheme for separating the data which is hidden in encrypted images and it can be reversible also. Heresome space is created to accommodate extra data in encrypting side and recovers the original data without any error in decrypting side [1]. Dr. P.V.Rama Raju, et.al. proposed a different technique which is used to hide gray images in RGB Image. In this the cover image is divided into sub images and by leaving RED gray scale image, embedding of gray scale image in remaining GREEN and BLUE gray scale images is explained [7]. V. S. Verma and R. K. Jha, are published paper on robust digital image watermarking, this paper presents a thorough literature review of how to avoid different categories of attacks with robust 
digital image watermarking applied in various types of images [8]. A. Upadhyay and M. Dave, are presented paper on color image watermarking for medical applications, in this paper they applied Discrete Wavelet Transform (DWT) up to the second level of decomposition as watermarking technique to the color images[11]. S. Rawat, and B. Raman, are published paper on combination of watermarking algorithm and cryptography techniques, in this paper they proposed how effectively embed a digital watermark in an image based on the fast discrete cosine transform (DCT) algorithm without disturbing the original image and explained how effectively encrypting is carried out [13]. R. O. Preda, et al. are presented paper on active image fake detection scheme based on a new watermarking scheme, in this paper they proposed wavelet packets based watermarking algorithm which are used to protect an image copyright [14]. N. Otsu, published paper on a selecting different threshold values from gray-level histograms, here different thresholding values are selected for picture segmentation and introduces non-parametric and new methods for segmentation [17]. G. Nagaraju, et al., are presented paper on encrypting different color images after hiding some gray images in it, this paper mainly focused on a key based steganography and a hiding the gray scale images by substitution of lower significant bits method [18]. S. Na, L. Xumin and G. Yong, are presented paper on a new approach to segmentation techniques, this paper discusses the shortcomings of standard k-means algorithm and briefly analyses those algorithms [19]. J. Wang and X. Su, are published paper on a new version of a segmentation algorithm, this paper introduces a new technique of k-means algorithm using noise data filter. Here noise data is generated. Then density-based detection methods are developed for segmentation using noise data[20]. Lu Ming presented a paper on image segmentation algorithm research and improvement, this paper proposes improved genetic algorithm in image segmentation [21].In Section II, we present the proposed scheme for watermarking generation, data embedding and extraction. The segmentation method used for tumor extraction is presented in Section III. Results and analysis are presented in Section IV. We finalize with conclusions in Section V. This proposed algorithm implemented using MATLAB software version MATLAB R2015a.

\section{Proposed Image Watermarking Scheme}

The block diagram of the proposed segmentation technique and watermarking procedure are shown in Figure 1. Our goal is to hide the patient's data into the medical image as a watermark without disturbing the original image quality.

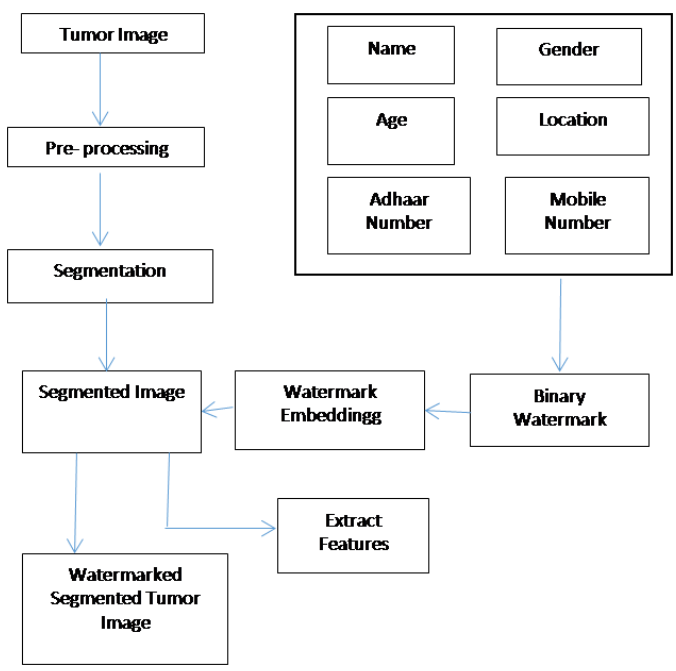

Fig. 1: Proposed watermark generation and data embedding scheme

\subsection{Watermark generation}

The data to be watermarked is the combination of six different patient's information fields like patient's gender (1 character), patient's age ( 2 characters), patient's name (50 characters), patient's location ( 25 characters), patient's ADHAAR number (12 characters) and patient's contact number (10 characters). These six components are converted into binary form and concatenated into a binary vector $w$ of 800 bits (100 characters). Each character will be having a unique 8 bit value generated by ASCII code. The generation of watermark using ASCII code with decimal values are shown in Table 1. This newly generated vector $\mathrm{w}$ is added with original image to obtain water marked image. To get original patient's information, at the decoder side inverse permutation will be performed.

Table 1: Alpha-numeric characters and symbols with their Decimal values

\begin{tabular}{|c|c|c|c|c|c|}
\hline Character & $\begin{array}{c}\text { Decimal } \\
\text { Value }\end{array}$ & Character & $\begin{array}{c}\text { Decimal } \\
\text { Value }\end{array}$ & Character & $\begin{array}{c}\text { Decimal } \\
\text { Value }\end{array}$ \\
\hline $\mathbf{A}$ & 03 & $\mathbf{a}$ & 56 & $\mathbf{0}$ & 186 \\
\hline B & 16 & b & 66 & 1 & 131 \\
\hline C & 71 & c & 77 & 2 & 37 \\
\hline D & 86 & d & 88 & 3 & 241 \\
\hline $\mathbf{E}$ & 42 & $\mathbf{e}$ & 166 & 4 & 47 \\
\hline $\mathbf{F}$ & 156 & f & 06 & 5 & 205 \\
\hline $\mathbf{G}$ & 05 & $\mathbf{g}$ & 53 & 6 & 112 \\
\hline H & 118 & h & 106 & 7 & 154 \\
\hline I & 08 & $\mathbf{i}$ & 222 & 8 & 200 \\
\hline $\mathbf{J}$ & 52 & $\mathbf{j}$ & 216 & 9 & 99 \\
\hline $\mathbf{K}$ & 30 & $\mathbf{k}$ & 11 & SPACE & 100 \\
\hline $\mathbf{L}$ & 21 & 1 & 176 & . & 48 \\
\hline M & 144 & $\mathbf{m}$ & 234 & , & 132 \\
\hline $\mathbf{N}$ & 93 & $\mathbf{n}$ & 136 & & \\
\hline $\mathbf{O}$ & 159 & $\mathbf{0}$ & 69 & & \\
\hline $\mathbf{P}$ & 14 & p & 210 & & \\
\hline Q & 104 & q & 55 & & \\
\hline $\mathbf{R}$ & 72 & $\mathbf{r}$ & 18 & & \\
\hline $\mathbf{S}$ & 81 & $\mathbf{s}$ & 26 & & \\
\hline $\mathbf{T}$ & 228 & $\mathbf{t}$ & 250 & & \\
\hline $\mathbf{U}$ & 149 & $\mathbf{u}$ & 190 & & \\
\hline V & 127 & $\mathbf{v}$ & 45 & & \\
\hline W & 246 & $\mathbf{w}$ & 246 & & \\
\hline $\mathbf{X}$ & 161 & $\mathbf{x}$ & 177 & & \\
\hline $\mathbf{Y}$ & 181 & $\mathbf{y}$ & 62 & & \\
\hline $\mathbf{Z}$ & 235 & $\mathbf{Z}$ & 126 & & \\
\hline
\end{tabular}

\subsection{Watermark embedding}

Let the segmented image be of size (200 200) pixels and the value of characters ' $w$ ' are to be embedded in a row or column of the segmented image by the data-hider. Hence, these values are kept in the first row (particular selection) of the segmented image. In this example, since the ' $w$ ' values are represented by 800 bits, and the first 100 pixels of the first row of the image are selected for location for embedding the data.

Then, the remaining pixels of the image are kept same. Then, the 8-bit binary values (each pixel) are converted to gray values and are arranged into their original positions. Combining these, the segmented image containing additional data is obtained with no change in size to the segmented image. Hence, additional data is embedded in a segmented image.

\subsection{Watermark extraction}

To get original patient's information, at the decoder side inverse permutation will be performed. Here the bits which embedded into the watermarked image are extracted. These bits are converted into 8 bit length bytes. These bytes are converted into characters by using ASCII code. Finally the patient's details are extracted. 


\section{Image Segmentation Algorithm}

Before going to apply segmentation, pre-processing of image is very important. For this convert original color image into gray scale image. And for contrast enhancement, applying different filtering methods like median filter, adaptive filter is carried out. Next applying morphological operations like erosion and dilation are carried out. Segmentation refers to the process of partitioning a digital image into multiple segments. Image segmentation is typically used to locate objects and boundaries in image. Image segmentation can also be considered as a process of assigning a label to every pixel in an image such that pixels with the same label share certain visual characteristics. It can also be defined as a technique which partitions a given image into a finite number of non- overlapping regions with respect to some characteristics, such as gray value distribution, texture. Segmentation subdivides an image into its likely regions or objects. After subdividing the image into different related likelihood clusters, most of the information of original image is elevated. Discontinuity and similarity are two basic properties of intensity values of the most segmentation algorithms.

In the first category the approach is based on abrupt changes in intensity, such as edges which are used to partition an image. The second category approaches is based on partitioning an image into regions that are similar according to a set of predefined criteria. In this paper second approach is used. i.e. K-means based Segmentation: In the year 1967 MacQueen developed the K-means algorithm. K-means is the clustering method which forms $\mathrm{k}$ clusters of $\mathrm{n}$ pixel objects, wherein each pixel object belongs to the cluster of the nearest mean. Given a set of observations (x1, x2 ... xn), where each observation is a d-dimensional real vector. Partition the $n$ observations into $\mathrm{k}$ number of sets where $(\mathrm{k} \leq \mathrm{n}) \mathrm{S}=\mathrm{S} 1, \mathrm{~S} 2, \ldots, \mathrm{Sk}$ is the main aim of the $\mathrm{K}$-means clustering, so as to minimize the within-cluster sum of squares, Where $\mathrm{Cj}$ is the mean of points in Si. $\mathrm{m}=\mathrm{xj} \varepsilon \mathrm{Si}$. It is a simple clustering method and gives fast outputs as well.

The k-means algorithm is an iterative technique that is used to partition an image into K clusters.

Algorithm for K means Clustering:

Step 1: Choose $\mathrm{K}$ centroids at random from input image.

Step 2: Partition the image into k clusters.

Step 3: Calculate the mean (centroid) of the k clusters for object $\mathrm{i}$ $=1 \ldots \mathrm{N}$. Repeat this until no reallocations occur.

Step 4: According to the clustered indexed Image separate image into $\mathrm{K}$ sub images.

Step 5: To extract exact tumor part from image, apply thresholding which is based on intensity and area. This algorithm is the general procedure for the perfect solution. The initial set of clusters and the value of $\mathrm{k}$ decides the quality of the solution.

\section{Experimental Results}

\section{Example 1}

Before going to apply segmentation, pre-processing of image is very important. Original tumor image and its gray-scale image are given in Figure 2. This original image is taken from internet and it is resized into $200 \mathrm{X} 200$ pixel image, and it is converted into gray scale image. With this 24 bit image is converted into 8 bit image.
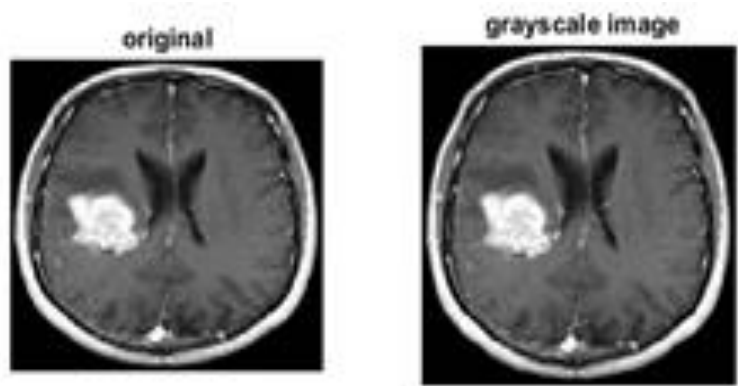

Fig. 2: Original image and its Gray-scale image

Applying different filters on image, the contrast and brightness enhancement is done and its results are shown in Figure 3.The median filter removes noise from an image or signal. Generally it is a nonlinear digital filtering technique which improves the results of edge detection on an image. Adaptive filtering is used to enhance or restore data by removing noise without disturbing the image.
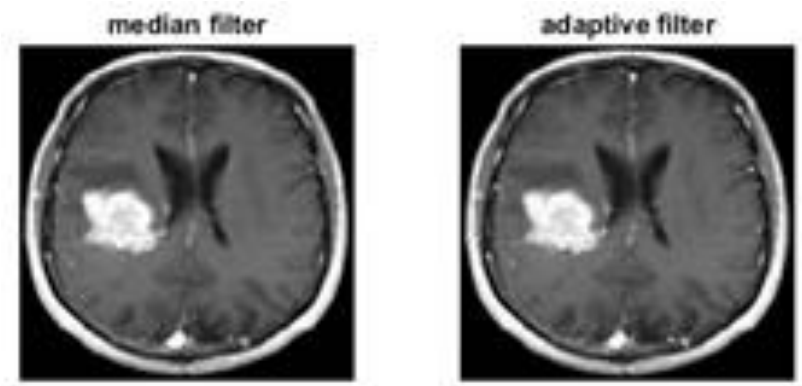

Fig. 3: Median filtered image and Adaptive filtered image

Applying morphological operations like erosion and dilation on image, the shapes of segmented area will be changed and its results are shown in figure 4. Dilation adds pixels to boundaries of area of interest and erosion sharpens the boundaries of area of interest. Here segmented part is the area of interest.
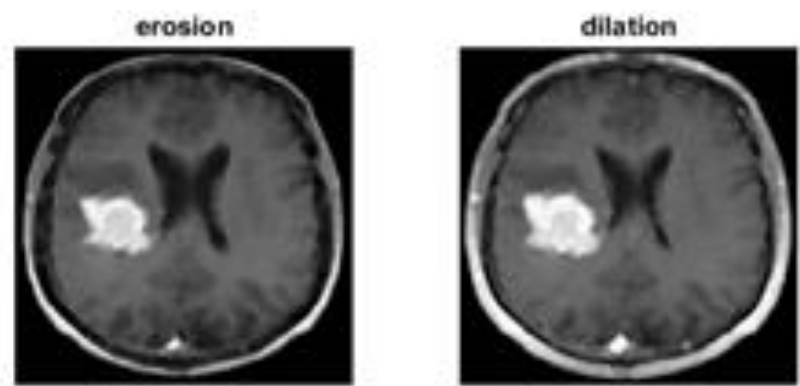

Fig. 4: Erosion image and Dilation image

After pre-processing, K-means clustering is used for segmentation. From segmented image, the below parameters are extracted using some pre-defined techniques.

\section{The Parameters}

Tumor Area $\left(\mathrm{mm}^{\wedge}{ }^{\wedge} 2\right)=153.4706$

Perimeter $(\mathrm{mm})=87.33$

Type of tumor: malignant.

To get watermarked image the below patient's details are embedded in segmented image. Here Patient's name, age, gender, location, ADHAAR number and contact number are embedded. These characters are converted into binary form by using ASCII code. This binary information is embedded into segmented image by replacing the existing binary values. The segmented image and 
resultant watermarked image after embedding the following data, are shown in Figure 5.

$\begin{array}{ll}\text { Gender: } & \text { M, } \\ \text { Age: } & 62, \\ \text { Name: } & \text { N. Apparao, } \\ \text { Location: } & \text { Gudivada, } \\ \text { ADHAAR number: } & 456789123852, \\ \text { Contact number: } & 9876543210\end{array}$
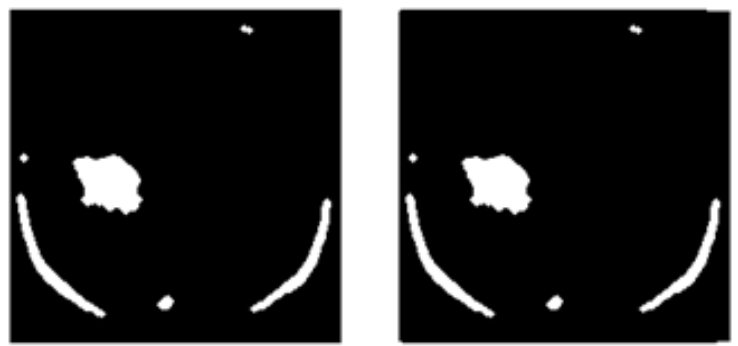

Fig. 5: Segmented image and Watermarked image

\section{Example 2}

Original tumor image and its gray-scale image are given in Figure 6. This original image is taken from internet and it is resized into 200 X 200 pixel image, and it is converted into gray scale image. With this 24 bit color image is converted into 8 bit gray scale image.
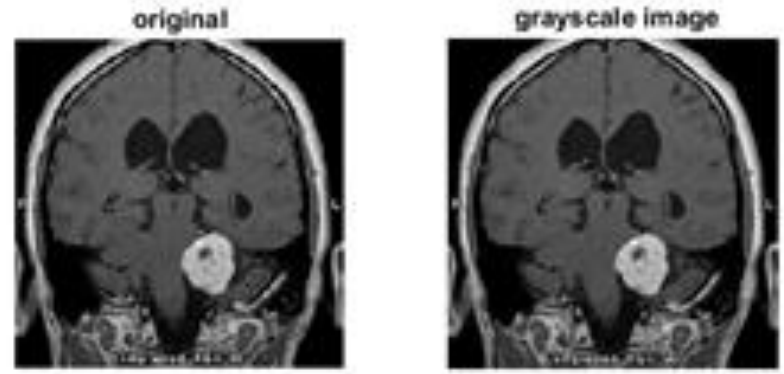

Fig. 6: Original image and its Gray-scale image

Applying different filters on image, the contrast and brightness enhancement is done and its results are shown in Figure 7. The median filter removes noise from an image or signal. Generally it is a nonlinear digital filtering technique which improves the results of edge detection on an image. Adaptive filtering is used to enhance or restore data by removing noise without disturbing the image.
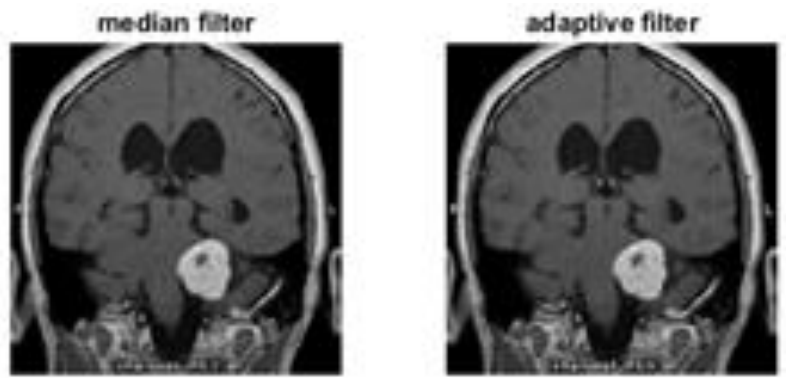

Figure 7. Median filtered image and Adaptive filtered image

Applying morphological operations like erosion and dilation on image, the shapes of segmented area will be changed and its results are shown in Figure 8. Dilation adds pixels to boundaries of area of interest and erosion sharpens the boundaries of area of interest. Here segmented part is the area of interest.
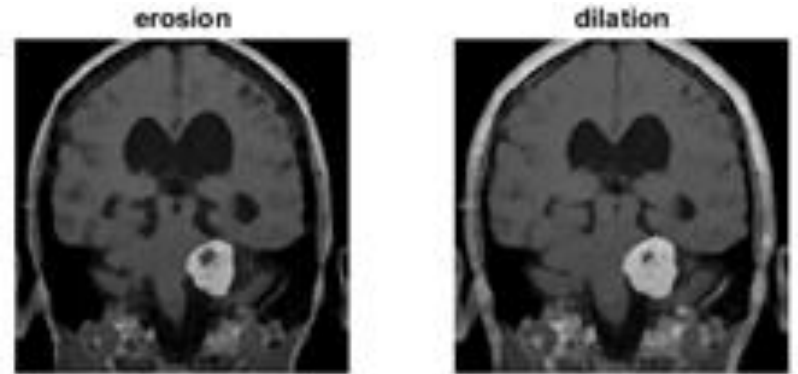

Fig. 8: Erosion image and Dilation image

After pre-processing, K-means clustering is used for segmentation. From segmented image, the below parameters are extracted using some pre-defined techniques.

\section{The Parameters}

Tumor Area $\left(\mathrm{mm} \cdot{ }^{\wedge} 2\right)=112.14$

Perimeter $(\mathrm{mm})=56.71$

Type of tumor: Benign.

To get watermarked image the below patient's details are embedded in segmented image. Here Patient's name, age, gender, location, ADHAAR number and contact number are embedded. These characters are converted into binary form by using ASCII code. This binary information is embedded into segmented image by replacing the existing binary values. The segmented image and resultant watermarked image after embedding the following data, are shown in Figure 9.

Gender:

Age:

Name:

Location:

48 ,

U. Rudrama Devi,

Location:

Visakhapatnam,

ADHAR number:

334296017742

Contact number: 8967498321.
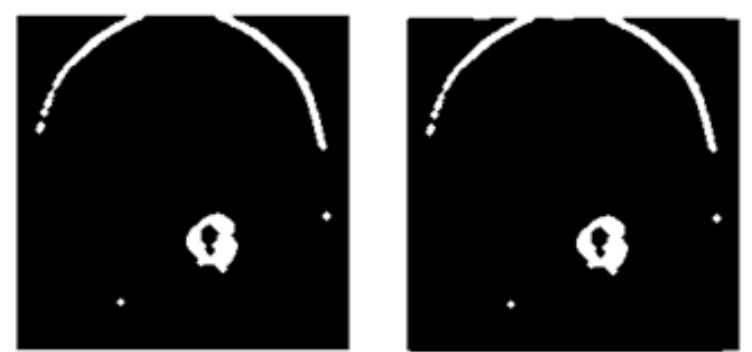

Fig. 9: Segmented image and Watermarked image

\section{Conclusions}

This is a simple and useful technique proposed to water mark the patient's details in brain tumor image. An Image watermark is the invisible image or data which may be inserted into digital image. The inserted watermark should not disturb or modify the visual quality of the tumor image and also it is proof against some signal processing operations, along with cropping, resizing, and some pre-processing techniques and segmentation. In this work, a brain tumor image is pre-processed and segmented and watermarked the patient data into the image. And also some features of segmented tumor image are calculated. Encrypting the final image with wellknown encryption algorithms is also possible to avoid unnecessary nuisance from information hackers. Add a unique ID tag to each encrypted image and exporting the encrypted image on to the cloud is very useful for doctors to verify the patient's details through internet. 


\section{References}

[1] Xinpeng Zhang, "Separable Reversible Data Hiding in Encrypted Image", IEEE Information Forensics and Security, Vol 7, No.2, April 2012.

[2] G. Nagaraju and T. V. Hyma Lakshmi, "Image Encryption using Secret-key images and Scan patterns", International Journal of Advances in Computer, Electrical \& Electronics Engineering., Vol. 2, Sp. Issue of NCIPA 2012, pp. 13-18.

[3] Chi-Kwong Chan \& L.M. Cheng (2001), "Improved Hiding Data in Images by Optimal Moderately Significant Bit Replacement", IEEE Electronics Letters, Vol. 37, No. 16, Pp.1017-1018.

[4] G. Naga Raju, James Vijay, "Secret-key based Separable Reversible Data-Hiding in Encrypted image," National Conference on VLSI, Signal processing \& Communications NCVSComs-2011.

[5] X.Zhang, "Reversible data hiding in encrypted image," IEEE Signal Processing. Lett vol. 18, no. 4, pp. 255-258, Apr. 2011

[6] Panduranga H.T, Naveenkumar S.K, "A novel image encryption method using 4 out of 8 code", proc.CommV'09, pp. 460-462, 2009.

[7] Dr. P.V.RamaRaju, T. Anvesh Gandhi, G. Naga Raju , "RGB Image Steganography using Zigzag Pixel Indicator and Scan Techniques"International Journal Of Research In Electronics And Computer Engineering., Vol. 3 Issue 3, July-Sept. 2015 ISSN: 23939028 (print), ISSN: 2348-2281 (online) Pp103-Pp107

[8] V. S. Verma and R. K. Jha, "An Overview of Robust Digital Image Watermarking”, IETE Technical Review, vol. 32, pp. 479-496, Nov 2015.

[9] S. A. Parah, J. A. Sheikh, U. I. Assad, and G. M. Bhat, "Realisation and robustness evaluation of a blind spatial domain watermarking technique", International Journal of Electronics, vol. 104, no. 4, pp. 659-672, 2017.

[10] Q. T. Su, Y. G. Niu, Q. J. Wang, and G. R. Sheng, "A blind color image watermarking based on DC component in the spatial domain," Optik, vol. 124, no. 23, pp. 6255-6260, 2013.

[11] A. Upadhyay and M. Dave, "Robust and Imperceptible Color Image Watermarking for Telemedicine Applications", 2016 IEEE International Conference on Computing, Communication and Automation, pp. 1104- 1109, 2016.

[12] S.-J. Horng, D. Rosiyadi, T. Li, et al., "A blind image copyright protection scheme for e-government", Journal of Visual Communicationand Image Representation, vol. 24, no. 4, pp. 1099-1105, 2013.

[13] S. Rawat, and B. Raman, "A blind watermarking algorithm based on fractional Fourier transform and visual cryptography", IEEE Signal Processing, vol. 92, no. 6, pp. 1480-1491, 2012.

[14] R. O. Preda, D. N. Vizireanu, S. Halunga, "Active image forgery detection scheme based on semi-fragile watermarking", Revue Roumaine des Sciences Techniques-SerieElectrotechnique et Energetique, Vol. 61, Issue 1, pp. 58-62, ISSN: 0035-4066, 2016.

[15] P. Cavalcanti, J. Scharcanski, L. Di Persia, and D. Milone, "An ICAbased method for the segmentation of pigmented skin lesions in macroscopic images," in Proc. IEEE Annu. Int. Conf. Eng. Med. Biol. Soc., pp. 5993-5996, 2011.

[16] Cavalcanti, P.; Scharcanski, J. A Coarse-to-Fine Approach for Segmenting Melanocytic Skin Lesions in Standard Camera Images. Computer Methods and Programs in Biomedicine, Vol. 112, No. 3 , pp. 684-93, 2013.

[17] N. Otsu, "A threshold selection method from gray-level histograms," IEEE Trans. Syst., Man, Cybern, vol. 9, no. 1, pp. 62-66, Jan. 1979.

[18] P. V. Ramaraju, G. N. Raju and P. R. Krishna, "Image encryption after hiding (IEAH) technique for color images," 2016 International Conference on Signal Processing, Communication, Power and Embedded System (SCOPES),Paralakhemundi, 2016, pp. 1202-1207. doi: 10.1109/SCOPES.2016.7955631

[19] S. Na, L. Xumin and G. Yong, "Research on k-means Clustering Algorithm: An Improved k-means Clustering Algorithm," 2010 Third International Symposium on Intelligent Information Technology and Security Informatics, Jinggangshan, 2010, pp. 63-67. doi: 10.1109/IITSI.2010.74

[20] J. Wang and X. Su, "An improved K-Means clustering algorithm," 2011 IEEE 3rd International Conference on Communication Software and Networks, Xi'an, 2011, pp. 44-46. doi: 10.1109/ICCSN.2011.6014384

[21] Lu Ming, "Image segmentation algorithm research and improvement," 2010 3rd International Conference on Advanced Computer Theory and Engineering (ICACTE), Chengdu, 2010, pp. V5-211V5-214. doi: 10.1109/ICACTE.2010.5579114 